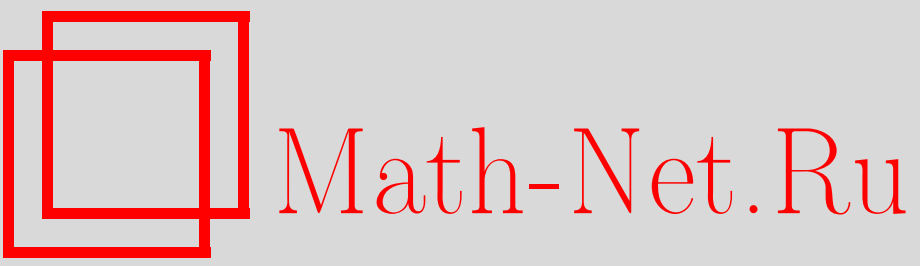

С. Б. Климентов, Об изгибаниях поверхностей рода $p \geq 1$ положительной внешней кривизны, Итоги науки и техн. Сер. Соврем. мат. и ее прил. Темат. обз., 2019, том 169, 17-22

DOI: https://doi.org/10.36535/0233-6723-2019-169-17-22

Использование Общероссийского математического портала Math-Net.Ru подразумевает, что вы прочитали и согласны с пользовательским соглашением

http://www. mathnet.ru/rus/agreement

Параметры загрузки :

IP: 54.89 .56 .158

26 апреля 2023 г., 16:10:13 


\title{
ОБ ИЗГИБАНИЯХ ПОВЕРХНОСТЕЙ РОДА $p \geqslant 1$ ПОЛОЖИТЕЛЬНОЙ ВНЕШНЕЙ КРИВИЗНЫ
}

\author{
(C) 2019 г. \\ С. Б. КЛИМЕНТОВ \\ Посвящается восъмидесятилетию Валентина Трофимовича Фоменко
}

\begin{abstract}
АннотАция. Статья представляет собой обзор работ по изгибаниям поверхностей положительной внешней кривизны рода $p \geqslant 1$.
\end{abstract}

Ключевые слова: изгибание поверхностей, кривизна, род поверхности.

\section{ON BENDING OF SURFACES OF GENUS $p \geqslant 1$ OF POSITIVE EXTERNAL CURVATURE}

\section{(c) 2019 S. B. KLIMENTOV}

AbStract. This paper is a review on bending of surfaces of positive external curvature of genus $p \geqslant 1$.

Keywords and phrases: surface bending, curvature, genus of a surface.

AMS Subject Classification: 53C40

1. Основные определения и обозначения. Обозначим через $M^{2}$ двумерное дифференцируемое многообразие (возможно, с краем) с дифференцируемой структурой класса $C_{\alpha}^{k}, k \geqslant 1$, $0 \leqslant \alpha \leqslant 1$ (обозначения функциональных пространств стандартные; см. [1]); $\left(u^{1}, u^{2}\right)$ - локальные координаты в некоторой (произвольной) координатной окрестности $U$. Будем всегда считать, что замыкание $\bar{U}$ компактно и содержится в некоторой координатной окрестности $V$ той же дифференцируемой структуры.

Обозначим через $g=g_{i j} d u^{i} d u^{j}$ риманову метрику на $M^{2}$ класса $C_{\alpha}^{k-1}$. Таким образом, $\left(M^{2}, g\right)-$ двумерное риманово многообразие класса $C_{\alpha}^{k-1}$. Аналогично определим трехмерное риманово многообразие (риманово пространство) $\left(R^{3}, G\right)$.

Под поверхностью $S$ класса $C_{\alpha}^{k}$ в $\left(R^{3}, G\right)$ будем понимать такое изометрическое погружение $f:\left(M^{2}, g\right) \rightarrow\left(R^{3}, G\right)$ класса $C_{\alpha}^{k}$, что $f\left(M^{2}\right)=S$. Там, где это не может вызвать недоразумений, будем отождествлять образ $f\left(M^{2}\right)=S$ с погружением $f$.

Две различные изометричные поверхности $S \neq S^{\prime}$ одной регулярности в $\left(R^{3}, G\right)$, вообще говоря, являются погружениями различных изометричных римановых многообразий. В случае регулярности $C_{\alpha}^{k}, k \geqslant 1,0<\alpha<1$, эти поверхности можно считать погружениями одного и того же риманова многообразия $\left(M^{2}, g\right)$; при этом соответствующие друг другу по изометрии точки на $S$ и $S^{\prime}$ будут иметь общий прообраз на $M^{2}$ (см. $[12,15]$.

В римановом пространстве, в котором имеется группа движений, поверхность $S$ называется однозначно определенной, если любая другая поверхность $S^{\prime}$, изометричная $S$, конгруэнтна $S$. Конгруэнтные поверхности называют тривиально изометричными. 
Будем считать риманово пространство $R^{3}$ евклидовым пространством $E^{3}$, а многообразие $M^{2}$ компактным. Зафиксируем на $M^{2}$ некоторое конечное покрытие координатными окрестностями $\left\{U_{i}\right\}_{i=1}^{n}$. Если вектор-функция $\boldsymbol{r}$ принадлежит классу $C_{\alpha}^{k}\left(M^{2}\right)$, определим ее норму следующим образом (см. [5]):

$$
\|\boldsymbol{r}\|_{C_{\alpha}^{k}\left(M^{2}\right)}=\max _{i=1, \ldots, n}\|\boldsymbol{r}\|_{C_{\alpha}^{k}\left(\bar{U}_{i}\right)} ;
$$

эта норма банахова. Аналогично для скалярных функций и тензорных полей соответствующей регулярности.

Деформацией класса $C^{m}, m \geqslant 0$, по параметру поверхности $S=f\left(M^{2}\right)$ будем называть такое отображение $f(t) \equiv f_{t}$ класса $C^{m}$ промежутка вещественной оси, содержащего нуль $([0,1],(-\varepsilon, \varepsilon)$, $\varepsilon>0$ и т. д.) в банахово пространство вектор-функций класса $C_{\alpha}^{k}\left(M^{2}\right)$, что

(1) $f(0) \equiv f_{0}=f\left(M^{2}\right)=S$;

(2) для любого $t$ вектор-функция $f_{t}$ задает погружение класса $C_{\alpha}^{k}$ многообразия $M^{2}$ в $E^{3}$.

Если для любого $t$ поверхность $S_{t}=f_{t}\left(M^{2}\right)$ изометрична поверхности $S=f_{0}\left(M^{2}\right)$, то деформация называется изгибанием класса $C^{m}$ по параметру. Если для любого $t$ изометрия тривиальна, изгибание называется тривиалъным. При отсутствии у поверхности нетривиальных изгибаний ее называют неизгибаемой.

Для некомпактных поверхностей определения аналогичны, только опираться приходится не на банахову структуру, а на структуру счетно-нормированного пространства Фреше.

Обобщения этих определений на случай риманова пространства несложны и используют некоторое фиксированное покрытие координатными компактными окрестностями уже и пространства $R^{3}$.

Ясно, что все характеристики деформируемой поверхности $S_{t}=f_{t}\left(M^{2}\right)$ будут функциями класса $C^{m}$ относительно параметра $t$, как-то: первый и второй основные тензоры (формы), гауссова и средняя кривизна и т. д. Если $\lambda=\lambda(t)$ - какая либо величина, связанная с поверхностью $S_{t}$, то вариацией функции $\lambda(t)$ называют выражение (если $m \geqslant 1$ )

$$
\delta \lambda=\left.\frac{d \lambda(t)}{d t}\right|_{t=0} .
$$

Если при деформации $S_{t}$ имеем $\delta \mathrm{I}=0$, где $\mathrm{I}=g_{i j} d u^{i} d u^{j}$ - первая квадратичная форма поверхности, то деформация называется бесконечно малым (б.м.) изгибанием поверхности $S$, а векторное поле $\delta f$ - полем бесконечного малого изгибания. Принято отождествлять б.м. изгибания с одинаковым полем $\delta f$ (см. [11]), т.е. б.м. изгибание фактически есть класс эквивалентности деформаций.

В случае наличия в $R^{3}$ движений б.м. изгибание называется тривиалъным, если $\delta f$ есть сужение на $S$ поля скоростей движения пространства $R^{3}$.

Поверхность, у которой отсутствуют нетривиальные б.м. изгибания, называется жесткой.

2. Замкнутые поверхности ненулевого рода и положительной внешней кривизны. На существование в некоторых римановых пространствах (достаточно сложной топологической структуры) замкнутых регулярных поверхностей рода $p \geqslant 1$ и положительной внешней кривизны указал А. В. Погорелов (см. [13], [14, гл. 6, § 12]). Он же доказал, что поле любого б.м. изгибания замкнутой поверхности рода $p=1$ и положительной внешней кривизны есть тождественный нуль при условии закрепления одной точки поверхности, и продемонстрировал на примере, что условие закрепления точки существенно. Класс регулярности пространства и поверхности при этом не фиксируется, но фактически предполагается $C^{k}, k \geqslant 4$.

В вышеупомянутых работах А. В. Погорелов сформулировал утверждение, что любое поле б.м. изгибания замкнутой поверхности рода $p>1$ положительной внешней кривизны тождественно равно нулю, и выдвинул задачу доказательства этого утверждения.

Дальнейшее развитие теории изгибаний поверхностей рода $p>0$ положительной внешней кривизны проводилось В. Т. Фоменко и его учениками: Е. В. Тюриковым, С. Б. Климентовым, Ю. П. Золотухиным. В [18] В. Т. Фоменко предложил для изучения изгибаний поверхностей ненулевого рода аппарат теории обобщенных аналитических функций на римановых поверхностях и 
доказал сформулированную А. В. Погореловым теорему о жесткости замкнутых поверхностей рода $p>1$ положительной внешней кривизны. В [19] В. Т. Фоменко также доказал неизгибаемость таких поверхностей в пространстве постоянной кривизны; в случае $p=1$ при условии закрепления одной точки поверхности. В обеих этих работах требования на регулярность $C_{\alpha}^{k}$, $k \geqslant 3,0<\alpha<1$.

Два следующие результата по замкнутым поверхностям принадлежат С. Б. Климентову.

1. В [21] была доказана неизгибаемость замкнутых поверхностей рода $p \geqslant 1$ положительной внешней кривизны уже без ограничений на кривизну пространства; в случае $p=1$ при условии закрепления одной точки поверхности (методом, отличным от метода работы [19]) в предположениях, что пространство и поверхность класса регулярности $C_{\alpha}^{k}, k \geqslant 5,0<\alpha<1$.

2. В [9] были получены необходимые и достаточные условия существования б.м. деформации замкнутой поверхности рода $p \geqslant 1$ положительной внешней кривизны с заданным б.м. изменением метрики. Требования на регулярность поверхности и пространства те же, что и в предыдущем случае.

Отметим, что из второго результата следует, что не всякая метрика на многообразии $M^{2}$, близкая к погружаемой в виде поверхности положительной внешней кривизны, также погружаема, в отличие от случая $p=0$ (см. [14, гл. $6, \S 7]$.

Замкнутые поверхности положительной внешней кривизны с разрезами исследовались Ю. П. Золотухиным в $[2,3]$. Сформулируем эти результаты подробнее.

Пусть $V^{3}$ - трехмерное риманово пространство постоянной кривизны, $S \subset V^{3}$ - двумерная замкнутая поверхность положительной внешней кривизны $K_{e} \geqslant$ const $>0$, рода $p \geqslant 0 ; S \in C_{\alpha}^{3}$, $0<\alpha<1$. Далее, пусть $\mathcal{L}=\bigcup_{k=0}^{m} \mathcal{L}_{k}$ - совокупность $m+1$ замкнутых или незамкнутых непересекающихся и не имеющих общих концов ляпуновских кривых на $S$. Если все кривые $\mathcal{L}_{k}$, $k=0,1, \ldots, m$, замкнуты, то будем предполагать, что контур $\mathcal{L}$ ограничивает на $S$ связную область $S^{+}$. На контуре $\mathcal{L}$ обычным образом задаем положительное направление обхода. Если контур $\mathcal{L}$ состоит из незамкнутых кривых $\mathcal{L}_{k}, k=0,1, \ldots, m$, с концами $\alpha_{k}, \beta_{k}$, то за положительное направление обхода принимаем обход от $\alpha_{k} \mathrm{~K} \beta_{k}$. В дальнейшем рассматриваем поверхность $S$ с разрезом $\mathcal{L}$ и различаем левый $\left(\mathcal{L}^{+}\right)$и правый $\left(\mathcal{L}^{-}\right)$берега разреза. Будем рассматривать изометрические преобразования поверхности $S$ с разрезами в классе $C_{\alpha}^{3}\left((S \backslash \mathcal{L}) \cup\left(\mathcal{L}^{+} \cup \mathcal{L}^{-}\right)\right)$.

Пусть $\tau$-касательная плоскость поверхности $S$ в точке $s$ (расположенная в соприкасающемся к $V^{3}$ евклидовом пространстве); $D$ - индикатриса внешней кривизны поверхности в точке $s$, расположенная в плоскости $\tau$. Далее, пусть $S^{*}$ - изометричная $S$ поверхность, $s^{*} \in S^{*}$ - точка, соответствующая по изометрии точке $s \in S$, и $D^{*}$ - индикатриса кривизны в точке $s^{*}$. Обозначим через $\nu^{*}$ угол между соответствующими главными направлениями $\omega$ и $\omega^{*}$ индикатрис $D$ и $D^{*}$, считая, что $0 \leqslant \nu^{*} \leqslant \pi / 2$. Главные кривизны в точках $s$ и $s^{*}$ по направлениям $\omega$ и $\omega^{*}$ обозначим $k_{1}$ и $k_{1}^{*}$. Изгибом поверхности $S^{*}$ в точке $s^{*}$ при изометрическом преобразовании назовем комплексное число $I(z)=\nu^{*}+i\left(k_{1}^{*}-k_{1}\right)$. В [3] поверхность $S$ называется однозначно определенной, если при любом изометрическом преобразовании этой поверхности $I(z) \equiv 0$.

Пусть поверхность $S^{*}$ при изометрическом преобразовании вдоль берегов разреза подчинена внешней связи

$$
\Delta k_{n l}^{+}=a_{11} \Delta k_{n l}^{-}+a_{12} \Delta \tau_{g l}^{-}, \quad \Delta \tau_{g l}^{+}=a_{21} \Delta k_{n l}^{-}+a_{22} \Delta \tau_{g l}^{-},
$$

где $\Delta k_{n l}^{ \pm}, \Delta \tau_{g l}^{ \pm}$- соответственно приращения нормальной кривизны и геодезического кручения вдоль берегов разреза в направлении $l ; l$-заданное вдоль контура $\mathcal{L}$ поле направлений класса $C_{\alpha}^{1}(\mathcal{L}) ; a_{i j}$ - заданные функции класса $C_{\alpha}^{1}(\mathcal{L})$. Предположим, что в концах незамкнутых кривых направление поля $l$ совпадает с предельным положением в этих точках поля касательных направлений контура $\mathcal{L}$ и в этих точках $a_{i j}=\delta_{i j}$ (символ Кронекера). Введем функцию $d=\operatorname{det}\left(a_{i j}\right)$ и при $d>0$ число

$$
\varkappa=\frac{1}{2 \pi} \sum_{k=0}^{m} \arg \left(a_{11}+a_{22}+i\left(a_{12}-a_{21}\right)\right)_{\mathcal{L}_{k}} .
$$

Приращения аргумента вычисляются при положительном обходе контура $\mathcal{L}$. 



Рис. 1

Теорема 1 (см. [3]). В пространстве $V^{3}$ поверхность $S$ рода $p \geqslant 0$ с разрезом $\mathcal{L}$, подчиненная внешней связи (1), является однозначно определенной, если $d>0$ всюду на $\mathcal{L} u \varkappa<4-4 p$.

Вопрос, существуют ли нетривиальные изометрические преобразования у поверхности $S$ рода $p \geqslant 1$ с незамкнутыми разрезами без каких-либо внешних условий в [3] не обсуждался и остается открытым по сей день. Следует отметить, что при $p=0$ пунктированный в двух точках регулярный овалоид положительной гауссовой кривизны $K \geqslant$ const $>0$ в евклидовом пространстве $E^{3}$ всегда допускает нетривиальные изометрические преобразования (см. [14, гл. $6, \S 11])$.

3. Поверхности ненулевого рода и положительной внешней кривизны с краем. Такого сорта поверхности существуют не только в римановых пространствах сложной топологической структуры, но и в трехмерном евклидовом пространстве. Существование поверхностей рода $p \geqslant 1$, положительной, отделенной от нуля гауссовой кривизны, с регулярными краями строго доказано в [10]; как могут выглядеть такие поверхности, демонстрирует рис. $1^{1}$.

Первый вопрос, который здесь возникает, существуют ли вообще нетривиальные изгибания и б.м. изгибания таких поверхностей? Ответ положительный и он вполне аналогичен ответу на этот же вопрос для односвязных, гомеоморфных кругу поверхностей положительной кривизны. Сформулируем его точнее.

Пусть $S=f\left(M^{2}\right) \subset R^{3}$ - компактная поверхность рода $p \geqslant 0$ с краем, строго положительной внешней кривизны $K_{e} \geqslant$ const $>0$. Считаем, что $S, R^{3} \in C_{\alpha}^{k}, k \geqslant 5,0<\alpha<1$. Вводя в окрестности каждой точки $P \in M^{2}$ сопряженно изотермическую систему координат $(u, v)$, в которой вторая основная форма поверхности $S$ имеет канонический вид (см. [1, гл. $2, \S 6]$ )

$$
\mathrm{II}=\Lambda(u, v)\left(d u^{2}+d v^{2}\right)>0,
$$

превратим $M^{2}$ в риманову поверхность с краем с локальной униформизирующей $z=u+i v$, $i^{2}=-1$ (см. [24, гл. 2, §1]).

В $[5,6,20]$ установлено, что всякой голоморфной на римановой поверхности $M^{2}$ функции $\Phi(z) \in C_{\alpha}^{k-2}\left(M^{2}\right)$ соответствует вполне определенное б.м. изгибание поверхности $S$, причем различным функциям $\Phi_{1}(z) \neq \Phi_{2}(z)$ соответствуют различные б.м. изгибания. Также каждому семейству $\left\{\Phi_{t}(z)\right\}_{t \in(-\varepsilon, \varepsilon)} \subset C_{\alpha}^{k-2}\left(M^{2}\right), \varepsilon>0$, голоморфных на римановой поверхности $M^{2}$ функций, класса $C^{m}, m \geqslant 0$, по параметру $t$, соответствует вполне определенное изгибание класса $C^{m}$ поверхности $S$.

\footnotetext{
${ }^{1}$ Рисунок выполнен Е. В. Тюриковым в 1975 г.
} 
Поскольку изгибаний и б.м. изгибаний у поверхности положительной кривизны с краем «много», естественной является задача об изгибаниях при различных внешних условиях, в том числе и краевых.

Различные краевые задачи для б.м. изгибаний довольно обстоятельно исследованы в $[2-4,6-8$, $16,17,22,23]$.

Рассматривалось краевое условие

$$
\lambda(s) \delta k_{n}+\mu(s) \delta \tau_{g}=\sigma(s), \quad s \in \mathcal{L}=\partial S,
$$

где $\lambda(s), \mu(s), \sigma(s)$ - наперед заданные функции точек края $\mathcal{L}, \delta k_{n}$ - вариация нормальной кривизны, а $\delta \tau_{g}$ - вариация геодезического кручения вдоль края в направлении края при б.м. изгибании (см. $[16,17])$, а также краевое условие обобщенного скольжения

$$
\boldsymbol{\xi}(s) \cdot \boldsymbol{l}(s)=\gamma(s), q q s \in \mathcal{L}=\partial S,
$$

где $\boldsymbol{\xi}(s)$ - поле б.м. изгибания на краю поверхности, $\boldsymbol{l}(s)$-заранее заданное векторное поле на крае $\mathcal{L}, \gamma(s)$ - заданная скалярная функция на $\mathcal{L}$, точка означает скалярное произведение (см. $[6,7])$.

Получены различные условия жесткости и нежесткости поверхности при рассматриваемых краевых условиях. По причине их громоздкости мы их здесь не воспроизводим.

Более сложный (нелинейный) случай конечных изгибаний изучен меньше; в $[3,22]$ установлены лишь достаточные условия отсутствия изгибаний в пространствах постоянной кривизны, в [10] показано, что изгибания могут быть со сколь угодно большим изменением второй основной формы (изгиба, см. п. 2) в фиксированной точке.

Отметим, что любой результат, касающийся конечных изгибаний класса $C^{m}, m \geqslant 1$, автоматически влечет за собой аналогичный результат для б.м. изгибаний (для линеаризованной задачи; см. [11, гл. 1]).

Приведем один новый, пока не опубликованный результат относительно достаточных условий однозначной определенности поверхности положительной гауссовой кривизны в трехмерном евклидовом пространстве (который вполне мог быть получен 40 лет назад).

K автору обратился И. Х. Сабитов с вопросом: будут ли конгруэнтны две изометричные, локально выпуклые, регулярные поверхности положительной гауссовой кривизны, с краями, если конгруэнтны края этих поверхностей? Для ограниченных выпуклых поверхностей с краями утвердительный ответ на этот вопрос следует из результатов А. В. Погорелова по однозначной определенности общих выпуклых поверхностей с краем (см. [14, гл. 3, § 7]). Для компактных поверхностей произвольного рода $p \geqslant 0$, положительной, отделенной от нуля гауссовой кривизны, с регулярными краями, верен более общий результат (см. [12]).

Теорема 2. Пусть $S$ и $S^{\prime}$ - изометричные поверхности класса $C_{\alpha}^{k}, k \geqslant 3,0<\alpha<1$, рода $p \geqslant 0$, гауссова кривизна которых удовлетворяет условию $K \geqslant k_{0}=$ const $>0$. Если края этих поверхностей $\Gamma=\partial S$ и $\Gamma^{\prime}=\partial S^{\prime}$ содержат соответствующие друг другу по изометрии конгруэнтные дуги $\gamma \subset \Gamma u \gamma^{\prime} \subset \Gamma^{\prime}$, то поверхности $S$ и $S^{\prime}$ конгруэнтны.

Очевидно, дуги $\gamma$ и $\gamma^{\prime}$ можно считать расположенными (частично или полностью) внутри поверхностей $S$ и $S^{\prime}$.

\section{СПИСОК ЛИТЕРАТУРЫ}

1. Векуа И. Н. Обобщенные аналитические функции. - М.: Физматгиз, 1959.

2. Золотухин Ю. П. Изгибания поверхностей положительной внешней кривизны с разрезами в римановом пространстве/ Дисс. на соиск. уч. степ. канд. физ.-мат. наук - Одесса, 1980.

3. Золотухин Ю. П. Об однозначной определенности поверхностей с разрезами в пространстве постоянной кривизны// Укр. геом. сб. - 1984. - 27. - С. 42-49.

4. Золотухин Ю. П. О жесткости поверхностей ненулевого рода с краем и разрезами в римановом пространстве при внешних связях// Изв. Сев.-Кав. центра высш. школы. Сер. Естеств. науки. - 1984. № 1. - C. $22-27$.

5. Климентов С. Б. Изгибания поверхностей рода $p \geqslant 0$ положительной внешней кривизны// Укр. геом. сб. $-1976 .-19 .-$ С. $37-56$. 
6. Климентов C. Б. Бесконечно малые изгибания поверхностей рода $p \geqslant 1$ положительной внешней кривизны с краевым условием обобщенного скольжения// Укр. геом. сб. - 1976. - 19. - С. 57-65.

7. Климентов С. Б. Изгибания поверхностей рода $p \geqslant 0$ положительной внешней кривизны в римановом пространстве/ Дисс. на соиск. уч. степ. канд. физ.-мат. наук - Одесса, 1976.

8. Климентов С. Б. О гладкости решений краевых задач теории изгибаний поверхностей рода $p \geqslant 0 / /$ Укр. геом. сб. - 1978. - 21. - С. 34-44.

9. Климентов С. Б. О деформациях замкнутых поверхностей рода $p \geqslant 1$ с заданным бесконечно малым изменением метрики// Мат. сб. - 1979. - 108, № 3. - С. 307-325.

10. Климентов С. Б. О степени изгибаемости поверхностей положительной кривизны// Укр. геом. сб. 1981. - № 24. - C. 39-52.

11. Климентов С. Б. Введение в теорию изгибаний. Двумерные поверхности в трехмерном евклидовом пространстве. - Ростов-на-Дону: Изд-во ЮФУ, 2014.

12. Климентов С. Б. Об однозначной определенности локально выпуклых поверхностей положительной кривизны рода $p \geqslant 0$ с краем// Сиб. мат. ж. - 2019. - 60, № 1. - С. 109-117.

13. Погорелов A. B. Некоторые вопросы геометрии в целом в римановом пространстве. - Харьков: Изд-во ХГУ, 1957.

14. Погорелов A. В. Внешняя геометрия выпуклых поверхностей. - М.: Наука, 1969.

15. Сабитов И. Х. К вопросу о гладкости изометрий// Сиб. мат. ж. - 1993. - 34, № 4. - С. $169-176$.

16. Тюриков E. B. Некоторые краевые задачи теории изгибаний поверхностей в пространстве Лобачевского/ Дисс. на соиск. уч. степ. канд. физ.-мат. наук. - Ростов-на-Дону, 1975.

17. Тюриков Е. В. О жесткости поверхностей рода $p \geqslant 1$ с краем, расположенных в пространстве Лобачевского // Сиб. мат. ж. - 1976. - 17, № 5. - С. 1129-1140.

18. Фоменко В. Т. О жесткости и однозначной определенности замкнутых поверхностей рода $p \geqslant 1$ в римановом пространстве// Докл. АН СССР. - 1973. - 213, № 1. - С. 45-48.

19. Фоменко B. Т. Об однозначной определенности замкнутых поверхностей рода $p \geqslant 1$ в пространстве постоянной кривизны// Мат. заметки. - 1974. - 16, № 3. - С. 441-445.

20. Фоменко B. Т., Климентов С. Б. Об изгибаниях поверхностей рода $p \geqslant 0$ положительной внешней кривизны в римановом пространстве// Докл. АН СССР. - 1976. - 227, № 5. - С. 1064-1066.

21. Фоменко В. Т., Климентов С. Б. Неизгибаемость замкнутых поверхностей рода $p \geqslant 1$ и положительной внешней кривизны// Мат. сб. - 1976. - 101, № 3. - С. 402-415.

22. Фоменко B. Т., Тюриков E. В. Исследование основных уравнений теории поверхностей положительной внешней кривизны, расположенных в пространстве Лобачевского// Изв. Сев.-Кав. центра высш. школы. Сер. Естеств. науки. - 1977. - № 3. - С. 3-7.

23. Фоменко В. Т., Золотухин Ю. П. Исследование некоторых классов двусторонних внешних связей теории изгибаний поверхностей// Изв. вузов. Мат. - 1984. - № 11. - С. 71-74.

24. Шиффер М., Спенсер Д. К. Функционалы на конечных римановых поверхностях. - М.: ИЛ, 1957.

Климентов Сергей Борисович

Южный федеральный университет, Ростов-на-Дону;

Южный математический институт Владикавказского научного центра РАН

и Правительства Республики Северная Осетия-Алания, Владикавказ

E-mail: sbklimentov@sfedu.ru 